\title{
MiR-20a-5p Negatively Regulates NR4A3 to Promote Metastasis in Bladder Cancer
}

\author{
Haibei Yang, Zhao Chen, and Zhiming Liu \\ Department of Urology, Qujing No. 1 People's Hospital, Qujing 655000, Yunnan, China \\ Correspondence should be addressed to Zhiming Liu; zhiming19850@163.com
}

Received 21 September 2021; Revised 20 October 2021; Accepted 22 October 2021; Published 9 December 2021

Academic Editor: Wei long Zhong

Copyright (C) 2021 Haibei Yang et al. This is an open access article distributed under the Creative Commons Attribution License, which permits unrestricted use, distribution, and reproduction in any medium, provided the original work is properly cited.

\begin{abstract}
Metastasis is the leading cause of death in cancer patients. Therefore, the prediction and treatment of metastasis are critical in improving the survival of patients with bladder cancer. In this study, we aimed to investigate the role of miR-20a-5p and NR4A3 in bladder cancer and the regulatory relationship between them. The high expression of miR-20a-5p in the bladder cancer (BCa) tissues and cells was determined by qRT-PCR. Exogenous miR-20a-5p overexpression promoted the proliferation, migration, and invasion of BCa cells. MiR-20a-5p inhibition inhibited the BCa cell proliferation, invasion, and migration. NR4A3 was proved to be the target gene of miR-20a-5p by the double luciferase reporter assay. In addition, the reduction of NR4A3 could promote the proliferation, invasion, and clonal formation of the bladder cancer cells 5637 and T24. NR4A3 overexpression could reverse the carcinogenic effect of miR-20a. We further confirmed that the oncogenic effect of miR-20a was achieved by promoting EMT in tumor cells. MiR-20a-5p promoted the growth and metastasis of the bladder cancer cells by inhibiting the expression of the tumor suppressor gene NR4A3 and played a carcinogenic role in BCa. Thus, miR-20a-5p may become a potential therapeutic target for BCa treatment.
\end{abstract}

\section{Introduction}

Bladder cancer is the most common tumor of the urinary system, and its morbidity and mortality rank first [1-4]. Bladder cancer is characterized by easy metastasis and recurrence, and early bladder cancer has no obvious symptoms $[5,6]$. Therefore, bladder tumor metastasis often occurs in many diagnosed patients, resulting in a poor prognosis $[7,8]$. Considering the deficiency of the existing diagnosis and treatment methods of bladder cancer, new diagnostic markers and effective treatment strategies are urgently needed [9-12]. Specific miRNAs with an abnormal expression in the bladder are closely related to the occurrence and development of bladder cancer and may be a new target for the treatment of bladder cancer.

MiRNA is a noncoding small molecule RNA with a length of approximately 19 to 25 nucleotides. It is highly conserved, sequential, and tissue-cell specific in the eukaryotes. Recent studies have shown that mature miRNAs can complement and pair with the $3^{\prime} \mathrm{UTR}$ of the target
mRNA using the "seed region" (2 to 7 nucleotides) and bind to the $5^{\prime} \mathrm{UTR}$ or ORF, thereby playing a regulatory role [13]. The regulatory mechanism of miRNA on mRNA is determined by the degree of complementarity, complementary base pairs, and other factors [14]. In 2002, Calin et al. [15] reported the deletion of a small genomic region of 13q14 in patients with chronic lymphocytic leukemia, including miR15a and miR-16-1 genes, and proved for the first time that miRNA abnormalities are closely related to tumors. MicroRNA-20a-5p (miR-20a-5p) is a common miRNA that is upregulated in bladder cancer and functions as an oncogene $[16,17]$. However, the biological role and exact mechanism of miR-20a-5p in bladder cancer development and progression are unclear.

NR4A3, also known as Nor-1, is a member of the orphan nuclear receptor family [18]. NR4A3 is a new tumor suppressor gene. Its expression is downregulated in a variety of tumors. The upregulated expression of NR4A3 can inhibit cancer cell invasion and metastasis. However, whether miR-20a-5p targets NR4A3 in bladder cancer progression is unknown. The 
members of this family are called "orphan" receptors because their endogenous ligands have not yet been identified, and they rely on physiological signals, including fatty acids, prostaglandins, growth factors, calcium, inflammatory factors, peptide hormones, and neurotransmitters [19]. Orphan receptors are activated and act on the target genes involved in the regulation of the cell cycle, apoptosis, inflammation, metabolism, DNA repair, and tumorigenesis. Literature reports that NR4A3 is a tumor suppressor gene in myeloid leukemia [20]. Reducing the expression of NR4A1 and NR4A3 genes can lead to bone marrow proliferative tumors in mice [21]. Studies on solid tumors showed that the expression level of nor- 1 in thyroid follicular carcinoma tissues was significantly lower than that in a follicular thyroid tumor [22].

In this study, bioinformatics analysis showed that NR4A3 is among the functional potential target genes of miR-20a-5p. The effects of miR-20a-5p on the proliferation and apoptosis of the human bladder cancer cells were analyzed, and the possible mechanisms were discussed.

\section{Methods}

2.1. Specimen Collection and Patient Information. Thirty cases of bladder cancer tissue and the corresponding paracancer tissue were collected from Qujing No.1 People's Hospital from December 2016 to January 2019. The cases included 19 females and 11 males in the age range of 35 years to 68 years. All tissues were obtained after radical cystectomy. None of the patients underwent radiotherapy, chemotherapy, or immunotherapy before surgical resection. The postoperative pathological diagnosis was bladder urothelial carcinoma. The pathological types of bladder cancer were independently diagnosed by two experienced pathologists. The histopathological grade of bladder cancer was classified according to WHO standards. Clinical staging was based on UICC-TNM criteria. All patients signed an informed consent, which was approved by the ethics committee of Qujing No. 1 People's Hospital.

2.2. Cell Culture. The bladder carcinoma cell lines (EJ, J82, T24, and 5637) and normal bladder epithelial cell line (SVHUC-1) were purchased from the American Type Culture Collection (ATCC, Manassas, VA, USA). The cell lines were tested and authenticated using the Cell ID System (Promega, Madison, WI, USA). All the cells involved in this experiment were cultured in the incubator with $5 \% \mathrm{CO}_{2}$ at $37^{\circ} \mathrm{C}$ with the culture medium containing 10\% fetal bovine serum (Gibco, Life Technologies). The medium for normal prostatic epithelial cells was a keratocyte seropoten-free medium containing $0.05 \mathrm{mg} / \mathrm{ml} \mathrm{BPE}$ and $5 \mathrm{ng} / \mathrm{ml}$ epidermal growth factor. The culture medium for bladder cancer cells was RPMI1640. The cells were inoculated into a 6-well plate or a 48-well plate before transfection and were transfected with Lipofectamine ${ }^{\mathrm{TM}} 2000$ when the cell density reached $80 \%$ on day 2 .

\subsection{Establishment of Tumor-Bearing Animal Model.} Lentiviruses were cotransfected with an enveloped plasmid into the cells with $80 \%$ fusion. The virus supernatant was collected after $72 \mathrm{~h}$. After centrifugation, the concentration was $70,000 \times \mathrm{g} / \mathrm{min}$, and the inoculation density was $2 \times 10^{4}$ cell suspensions/mL. Polybrene, an infection-promoting reagent, was added and observed under a microscope after transfection for $36 \mathrm{~h}$. The cells at logarithmic growth stage were prepared into $5 \times 10^{7} / \mathrm{ml}$ cell suspension with $\mathrm{PBS}$. Twelve BALB/C nude mice were arbitrarily selected and divided into two groups with six mice in each group. The dose per inoculation site was $100 \mu \mathrm{L}$. The BALB/C nude mice were disinfected with iodophor and inoculated into the ipsilateral forelimbs and armpits. After inoculation, the nude mice were observed for 3 weeks. They were euthanized using carbon dioxide. The nude mice were put into the euthanasia chamber. The filling rate is $20 \% \mathrm{CO}_{2}$ per minute of the chamber volume. After 10 minutes, the nude mice were observed, found to lack breath, and were found to have faded eyes. Keep the carbon dioxide for 3 minutes after breathing stops. Make sure all nude mice die. The tumor tissue was stripped and weighed. The lung tissue was separated and fixed routinely.

2.4. Hematoxylin-Eosin (HE) Staining of Lung Tissue. The lung tissue was fixed in $10 \%$ formaldehyde. A conventional paraffin section was prepared. The slices were stained with hematoxylin-eosin (HE). The slices were immersed in xylene I and II for $15 \mathrm{~min}$ each, after which they were soaked in anhydrous ethanol I and II for $5 \mathrm{~min}$, respectively. Then, they were soaked in the following: $95 \%, 70 \%$, and $50 \%$ ethanol for $3 \mathrm{~min}$ each, and distilled water for $5 \mathrm{~min}$. The $\mathrm{HE}$ stain was added for $30 \mathrm{~s}$. Then, gentle rinsing with water was performed to revert to blue. The slices were sequentially soaked in $50 \%, 70 \%$, and $95 \%$ ethanol for 3 min for dehydration. The HE staining was added, followed by immersion in anhydrous ethanol for $5 \mathrm{~min}$ and transparent xylene I and II for $15 \mathrm{~min}$ each.

2.5. Cell Transfection. The miR-20a-5p mimics, miR-20a-5p inhibitor, NR4A3 small interfering RNA (si-NR4A3), vector-NR4A3, and their corresponding controls were purchased from Shanghai Jima Pharmaceutical Technology Co., LTD. The 5637 and T24 cells were inoculated into a 6-well plate. The cells with a density of up to $60 \%$ were transfected with the Lipofectamine ${ }^{\mathrm{TM}} 2000$ (Life Technologies, USA) according to the instructions. The fluid was changed after $6 \mathrm{~h}$, and transfection was performed for $48 \mathrm{~h}$.

2.6. QRT-PCR. MiRNAs were extracted from the tissues and cells according to the instructions of the miRNA extraction kit. The concentration and mass of the extracted RNA were determined using NanoDrop 1000 (Thermo Scientific, USA). RNA (500 ng) was used for the reverse transcription reaction. For the reverse transcription reaction of miRNA, the specific reverse transcription primer of miR-20a was used. After the cDNA product was diluted by five times, $2 \mu \mathrm{L}$ was used for the PCR reaction. The reaction system was as follows: cDNA at $2 \mu \mathrm{L}(100 \mathrm{ng}), 1 \mu \mathrm{L}$ for each upstream and downstream primer, $2 \times$ SYBR Green Mix (Life Technologies, 
USA) at $10 \mu \mathrm{L}$, and $6 \mu \mathrm{L}$ for sterile distilled water. The results were performed on ABI 7300 (ABI, Foster City, CA, USA) real time quantitative $\mathrm{PCR}$ instrument. The reaction conditions were as follows: $95^{\circ} \mathrm{C}$ for $30 \mathrm{~s}$, followed by $95^{\circ} \mathrm{C}$ for $30 \mathrm{~s}, 58^{\circ} \mathrm{C}$ for $30 \mathrm{~s}$, and $72^{\circ} \mathrm{C}$ for $30 \mathrm{~s}, 40$ cycles. The sequence used in this experiment was as follows: E-cadherin, F: $5^{\prime}$-CGCATTGCCACATACAC-3', R: 5'-CCTTCCATGACAGACCC-3'. Vimentin, F: $5^{\prime}$-ATGGCTCGTCACCTTCG- $3^{\prime}, \quad$ R: $5^{\prime}$ AGTTTCGTTGATAACCTGTCC- ${ }^{\prime}$. F: $\quad 5^{\prime}$-GGGAC CTGACTGACTACCTC-3', R: 5' $^{\prime}$-ACGAGACCACC TTCAACTCCAC-3'. U6, F: 5' -CTCGCTTCGGCAGCACATATACT-3'， R: $5^{\prime}$-ACGCTTCACGAATTTGCGTGTC- $3^{\prime}$. $\mathrm{U} 6$ and $\beta$-actin were used as the internal reference. $2^{-\Delta \Delta \mathrm{Ct}}$ methods were used to calculate the relative expression of the gene.

2.7. CCK-8 Assay. The cells in each group were inoculated in the 96-well plates with 3000 cells per well. After incubation for $48 \mathrm{~h}, 10 \mu \mathrm{L}$ of CCK- 8 reagent (Beyotime, Shanghai, China) was added to each well, and incubation was performed for $2 \mathrm{~h}$. The absorbance value of $450 \mathrm{~nm}$ (A value) was detected by the microplate reader (MultiskanEX, Lab Systems, Helsinki, Finland) and zeroized with a blank hole. The results were expressed as cell survival rate $=(\mathrm{A}$ value of control group/A value of experimental group $) \times 100 \%$.

2.8. Cell Migration Assay. The cell density was adjusted, and the cells were inoculated in the new 6-well plate. The cells were transfected with different plasmids when the fusion reached approximately $75 \%$ to $85 \%$. The sterile $10 \mu \mathrm{L}$ spear was used to cross the lines on the cell surface in the ultraclean table when the 6-hole plate was completely covered with cells. The cells were washed with PBS, fresh medium was added, and the culture was continued. Pictures were taken under an inverted microscope at 0 and $24 \mathrm{~h}$ after marking (Nikon, Japan).

2.9. Transwell. Matrigel and DMEM medium were diluted in the ratio of $1: 3$ to make the matrigel diluent. The diluent at $50 \mu \mathrm{L}$ was added to the Transwell membrane. Incubation was performed at $37^{\circ} \mathrm{C}$ for $30 \mathrm{~min}$ for solidification. The cell suspension $(100 \mu \mathrm{L})$ was added to the Transwell upper chamber. The medium containing $20 \%$ fetal bovine serum at $600 \mu \mathrm{L}$ was added to the Transwell assay. The cells were cultured for $24 \mathrm{~h}$ and removed. The cells were fixed with $10 \%$ paraformaldehyde for $15 \mathrm{~min}$. Crystal violet was used for staining for $15 \mathrm{~min}$. Five fields were observed under the microscope (Nikon, Japan) and randomly selected for drawing and counting.

2.10. Double Luciferase Reporting Assay. TargetScan software (http://www.targetscan.org/) was used to predict the target genes of miR-20a-5p. The $3^{\prime}$ UTR terminus of NR4A3 had complementary binding sites with miR-20a-5p. Mutant luciferase reporter vector (MUT) and wild-type luciferase reporter vector (WT) were constructed by mutating the $3^{\prime}$ UTR terminal complementary binding site of NR4A3. The binding sites were mutated by the quick-change site-directed mutagenesis kit (Agilent Technologies, Santa Clara, CA, USA). The double luciferase reporter gene was used (Promega, Madison, WI, USA). The construction of NR4A3$3^{\prime}$ UTR reporter vectors containing miR-20a- $5 \mathrm{p}$ binding sites (WT-NR4A3) and mutated mutator (MUT-NR4A3) were provided by Nanjing Novozyme Biotechnology Co., LTD. The miR-20a-5p mimics and miR-NC were cotransfected with WT-NR4A3 or MUT-NR4A3 to 5637 and T24 cells, respectively. The luciferase activity of the two groups of cells was detected by a dual luciferase reporter gene detection system (Promega, Madison, WI, USA) for $48 \mathrm{~h}$ after transfection.

2.11. Colony Formation Assay. A colony formation assay was performed to detect the ability of the cell clone formation. The cell count at the logarithmic phase was obtained, and the number of cells was adjusted. The cells were inoculated into 6-well plates with 1000 cells per well. The cells were cultured at $37^{\circ} \mathrm{C}$ and $5 \% \mathrm{CO}_{2}$ until colony formation. Three multiple holes are set in each group. The culture medium was removed after $48 \mathrm{~h}$. The cells were fixed with $40 \mathrm{~g} / \mathrm{L}$ of paraformaldehyde for $15 \mathrm{~min}$. Paraformaldehyde was removed, and the crystal violet dye solution was added for re-dyeing for $10 \mathrm{~min}$. The number of colony formation per hole was counted under the microscope (Nikon, Japan).

2.12. Immunofluorescence Staining. All treated cells were cultured on $18 \mathrm{~mm}$ cover glass slides for $24 \mathrm{~h}$. After the cells were completely adherent, they were fixed with $4 \%$ paraformaldehyde and sealed with 3\% BSA. The primary antibodies, including E-cadherin (Abcam, 1:200 dilution) and Vimentin (Abcam, 1:100 dilution), were added, and the cells were incubated overnight at $4^{\circ} \mathrm{C}$. After the cells were washed with PBS, they were incubated with AlexaFluor 488 or AlexaFluor 594 secondary antibodies (1:500 dilution). The nuclei were stained with 4, 6-diamidine-2-phenylindole, and the slides were observed under a laser scanning confocal microscope (Nikon, Japan).

2.13. Statistical Analysis. The experimental data were analyzed by SPSS 21.0 software, and the measurement data were expressed by the mean standard deviation $(\bar{x} \pm s)$ of three biological replicates or samples. A comparison between the two groups of data was conducted using a $t$-test. The differences in the mean of multiple groups were compared using a one-way ANOVA followed by Tukey's multiple comparison test. Spearman was adopted for correlation analysis. $P<0.05$ was considered statistically significant.

\section{Results}

3.1. MiR-20a-5p Was Upregulated in Bladder Cancer Tissues. To investigate the role of miR-20a-5p in bladder cancer, we collected 30 cases of bladder cancer tissues and paracancer control tissues that had been surgically removed. The expression of miR-20a-5p in bladder cancer and adjacent 
tissues was detected by qPCR. The experimental results showed that miR-20a-5p expression level in bladder cancer tissues was significantly higher than in adjacent control tissues (Figure 1(a)). We further measured the expression of miR-20a-5p in SV-HUC-1 and bladder cancer cells (EJ, T24, 5637, and J82) in normal bladder epithelial cells. Compared with SV-HUC-1 cells, the miR-20a-5p expression level in the bladder cancer cells increased and was the highest in 5637 and T24 cells (Figure 1(b)). Therefore, 5627 and T24 cells were selected for subsequent functional experiments.

\subsection{MiR-20a-5p Overexpression Promoted the Malignant} Progression of Bladder Cancer Cells 5637 and T24. To study the effects of miR-20a-5p on the biological functions of the bladder cancer cells, we overexpressed and reduced miR$20 \mathrm{a}-5 \mathrm{p}$ to detect the changes in the proliferation, invasion, migration, and clonal formation of the bladder cancer cells. The experiment was divided into four groups, namely mimics-NC, miR-20a-5p mimics, inhibitor-NC, and miR20a-5p inhibitor. Figure 2(a) shows the expression efficiency of miR-20a-5p in the 5637 cells. The experimental results showed that miR-20a-5p mimics can significantly upregulate miR-20a-5p expression. Similarly, the miR-20a-5p inhibitor inhibited miR-20a-5p expression. MiR-20a-5p expression level and efficiency in T24 were consistent with those in 5637 cells (Figure 2(b)). The proliferation detection results of 5637 and T24 cells showed that miR-20a-5p overexpression promoted the proliferation of the bladder cancer cells, whereas the miR-20a-5p inhibition decreased the proliferation of the bladder cancer cells (Figures 2(c) and 2(d)). The results of the 5637 and T24 cell invasion assay showed that miR-20a-5p overexpression promoted the invasion ability of the bladder cancer cells, whereas miR-20a-5p inhibition decreased the invasion ability of the bladder cancer cells (Figure 2(e)). The results of the cell migration detection of the 5637 and T24 showed that miR-20a-5p overexpression promoted the migration ability of the bladder cancer cells, whereas miR-20a-5p inhibition decreased the migration ability of the bladder cancer cells (Figure 2(f)). The results of proliferation and cloning of the 5637 and T24 cells showed that miR-20a-5p overexpression promoted the cloning and formation of the bladder cancer cells, whereas miR-20a-5p inhibition decreased the cloning and formation of the bladder cancer cells (Figure 2(g)).

\subsection{MiR-20a-5p Overexpression Promoted EMT in Bladder} Cancer. MiR-20a-5p can promote the invasion and migration of the bladder cancer cells by experiments. EMT is an important pathway for the invasion and metastasis of tumor cells, and hence, we detected the changes of the epithelial marker E-cadherin and the mesenchymal marker Vimentin. The immunofluorescence staining of the 5376 cell results showed that miR-20a-5p overexpression inhibited the E-cadherin expression, whereas E-cadherin expression was upregulated by miR-20a-5p (Figure 3(a)). The result of RTqPCR analysis in the 5376 cells was consistent with the above-mentioned phenomenon, which indicated that miR20a-5p overexpression promoted Vimentin expression, whereas miR-20a-5 inhibition downregulated Vimentin expression. The results from the T24 cells were consistent with those from the 5637 cells (Figure 3(c)). We performed animal experiments to verify the effect of miR-20a. Animal experiment results showed that miR-20a could promote tumor proliferation and metastasis (Figures 3(d) and 3(e)).

3.4. NR4A3 Was Targeted by miR-20a-5p. To study the downstream binding target genes of miR-20a-5p, we made predictions using the TargetScan website. The predicted results showed that miR-20a-5p could bind NR4A3 (Figure 4(a)). The dual luciferase reporter gene assay further demonstrated the binding of miR-20a-5p to NR4A3 (Figure 4(b)). The test results showed that miR-20a-5p mimics had inhibitory activity on the promoter of the wild NR4A3 but had no inhibitory effect on NR4A3 after mutation. Furthermore, we detected the effect of miR-20a-5p on NR4A3 expression. The experimental results showed that miR-20a-5p overexpression inhibited NR4A3 expression (Figure 4(c)). The detection results of the T24 cells were consistent with those of the 5637 cells. MiR-20a-5p overexpression inhibited NR4A3 expression, whereas miR20a-5p inhibition upregulated NR4A3 expression (Figure $4(\mathrm{~d})$ ). The results of NR4A3 expression in the bladder cancer showed that NR4A3 was highly expressed in the tissues of patients with bladder cancer compared with the paracancer control group (Figure 4(e)). We further analyzed the expression of NR4A3 using the UALCAN website that contains TCGA data. UALCAN (http://ualcan.path.uab.edu/index.html) is an effective cancer data online analysis and mining site, mainly based on the TCGA related cancer data in the database for analysis. The results also showed that NR4A3 was significantly downregulated in bladder cancer (Figure S1). The correlation between miR-20a-5p and NR4A3 showed that miR-20a-5p expression was negatively correlated with that of NR4A3 (Figure 4(f)). The above-mentioned results indicated that NR4A3 was the target gene of miR-20a-5p.

3.5. Reduction of NR4A3 Promoted Malignant Progression of Bladder Cells. To study the role of NR4A3, we used siRNA to knock down NR4A3. The experimental results showed that si-NR4A3 could effectively reduce NR4A3 expression (Figure 5(a)). The detection results of the T24 cells were consistent with those of the 5637 cells (Figure 5(b)). The effect of NR4A3 on the bladder cell proliferation was detected by CCK-8. Cell proliferation ability was inhibited after NR4A3 was knocked down (Figures 5(c) and 5(d)). The results of cell Transwell experiment showed that the invasive ability of the cells decreased after NR4A3 expression was knocked down (Figure 5(e)). The test results of the cell cloning ability of the 5637 cells showed that the cell cloning ability was enhanced when the expression level of NR4A3 was reduced. The results of the T24 cell cloning formation experiment were consistent with those of the 5637 cells. After the inhibition of NR4A3, the ability of cell cloning formation was enhanced (Figure 5(f)). These results indicate that NR4A3 plays the role of a tumor suppressor gene in bladder cancer. 


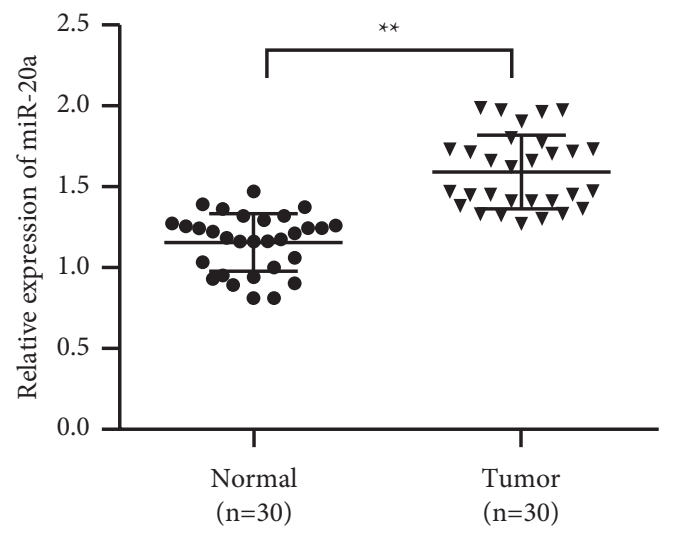

(a)

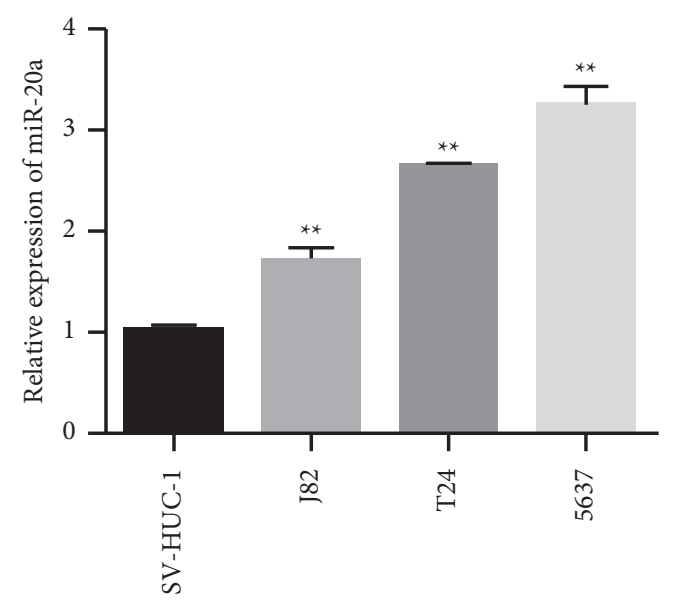

(b)

Figure 1: MiR-20a-5p expression is upregulated in bladder cancer. (a) MiR-20a-5p expression level detection. (b) Expression of miR-20a-5p in human bladder epithelial immortalized cells (SV-HUC-1) and bladder cancer cells (EJ, T24, 5637, and J82). ${ }^{* *} P<0.01$.

3.6. NR4A3 Overexpression Reversed the Carcinogenic Effect of MiR-20a-5p. To verify the relationship between NR4A3 and miR-20a-5p, we simultaneously transfected NR4A3 plasmids and miR-20a-5p mimics into the bladder cancer cells. The results of NR4A3 expression in 5637 cells and T24 showed that the NR4A3 overexpression plasmid could effectively upregulate NR4A3 expression, whereas miR-20a-5p mimics inhibited NR4A3 expression (Figures 6(a) and 6(b)). The cell proliferation detection results showed that NR4A3 overexpression inhibited the proliferation of the bladder cancer cells. The miR-20a-5p mimics promoted the proliferation of the bladder cancer cells, whereas NR4A3 could reverse the proliferation of miR-20a-5p (Figures 6(c) and 6(d)). Transwell test results showed that NR4A3 overexpression inhibited the invasion ability of the bladder cancer cells, and the miR-20a-5p mimics promoted the invasion ability of the bladder cancer cells, whereas NR4A3 could reverse the invasion ability of miR-20a-5p (Figures 6(e) and 6(f)). The results of the cell E-cadherin expression detection showed that NR4A3 overexpression promoted the expression of E-cadherin and that the miR20a-5p mimics inhibited E-cadherin expression. By contrast, NR4A3 could reverse the EMT-promoting effect of miR20a-5p (Figures 6(g) and 6(h)). The results of cell Vimentin expression detection showed that NR4A3 overexpression inhibited Vimentin expression and miR-20a-5p mimics upregulated Vimentin expression, whereas NR4A3 could reverse the EMT-promoting effect of miR-20a-5p (Figures 6(i) and 6(j)). The above-mentioned results showed that the overexpression of NR4A3 could reverse the carcinogenic effect of miR-20a-5p.

\section{Discussion}

In recent years, the abnormal expression of miRNA in tumors and its role in carcinogenesis have been gradually elucidated, providing important theoretical bases for the occurrence and development of tumors [23, 24]. In 2007, Gottardo et al. [25] first reported the miRNA expression profile in bladder cancer. Catto et al. [26] studied the expression of miRNAs in six bladder cancer cell lines, 20 normal bladder tissues, and 52 bladder cancer tissues. They found 16 abnormal miRNAs in bladder cancer tissues. However, no detailed explanation on whether the abnormal miRNA expression of these 16 was upregulated or downregulated was provided $[27,28]$.

MiRNA is closely related to bladder cancer occurrence and development. Based on their functions, miRNAs can be divided into oncogenes and tumor suppressor genes, which play important roles in the occurrence and development of bladder cancer [29]. The collection of miRNA biological specimens can provide valuable evidence for bladder cancer tumor formation. Therefore, the abnormal expression of one or more miRNAs may improve the risk stratification of patients and complement the histologic diagnosis of urinary tumors, especially in bladder cancer diagnosis. The miRNA with an oncogene function in the bladder cancer is a class of genes that can cause cell canceration, and the products encoded by such genes are related to tumor transformation. Thirty-three kinds of miRNA expression were upregulated in bladder cancer tissues, including miRNA-708, miRNA200b/c, miRNA-205, miRNA-182, miRNA-183, miRNA21, miRNA-96, miRNA17-5p, and miRNA-20a [30]. The results of this study are consistent with those of other studies. For example, miR-20a can promote the invasion and metastasis of the gallbladder cancer cells [31]. miR-20a increases the ability of the osteosarcoma cells to metastasize by regulating the expression of the Fas gene [32]. This study found that the overexpression of miR-20a can promote the proliferation, invasion, and migration of the bladder cancer cells. MiR-20a overexpression is also associated with the clonal formation of the bladder cancer cells $[33,34]$.

NR4A receptor plays an important role in the maintenance of cell homeostasis and disease occurrence and has attracted the interest of different researchers in various fields in recent years $[35,36]$. The NGFI-B family of orphan nuclear receptors includes three members NR4A1 (Nur77), NR4A2 (Nurr1), and NR4A3 (Nor1). They share similar 


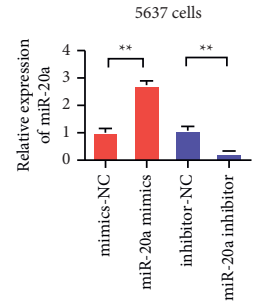

(a)

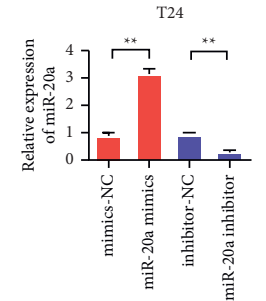

(b)

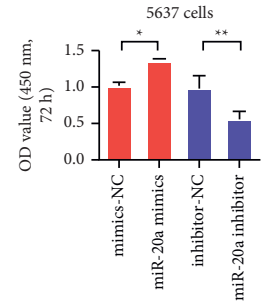

(c)

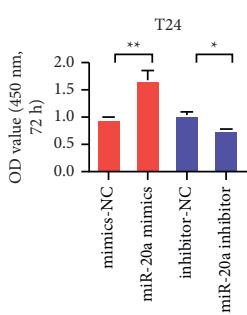

(d)

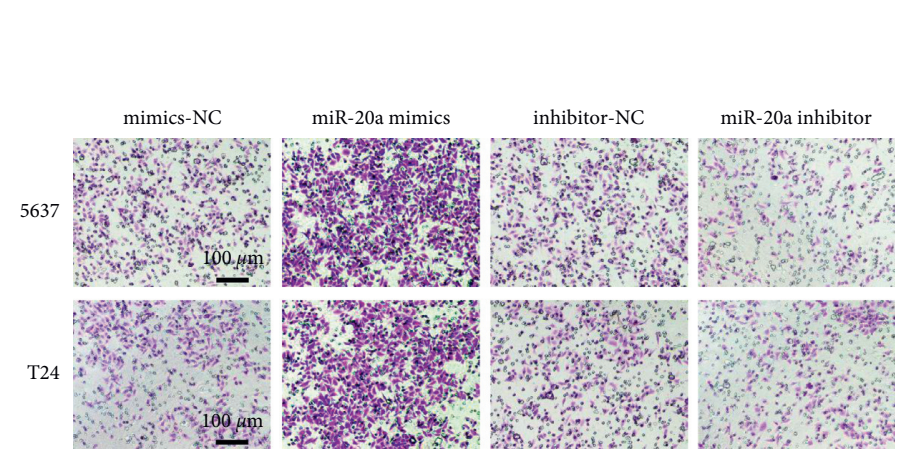

(e)
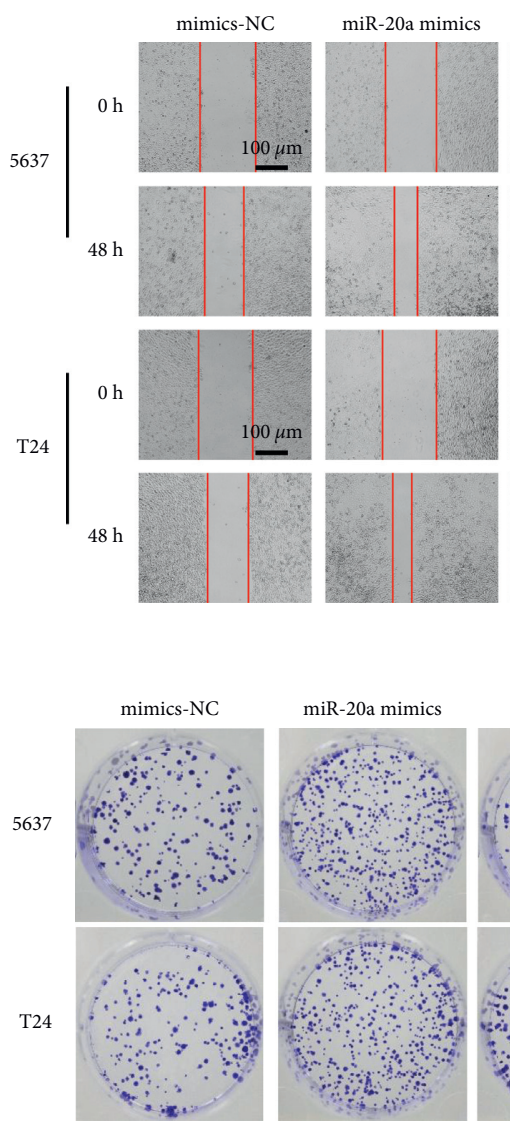

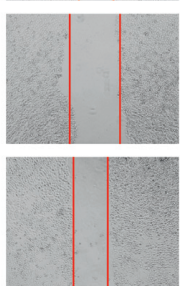

(f)
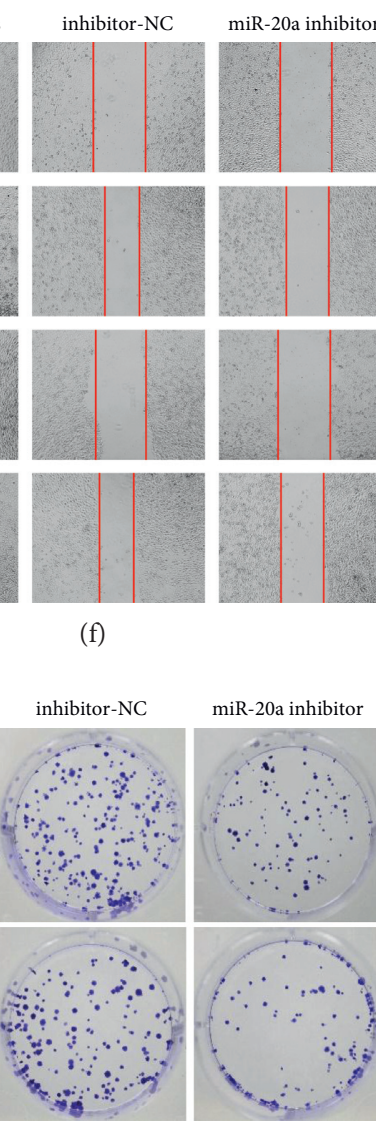

(g)

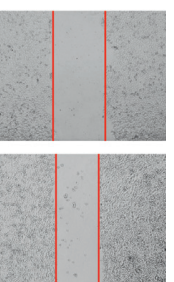

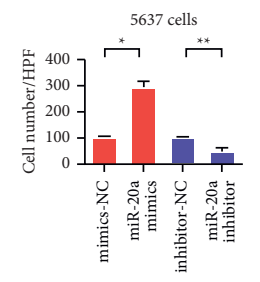

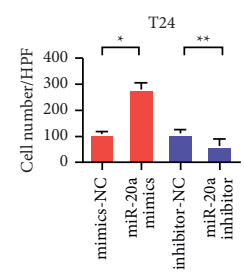

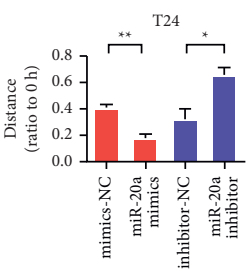

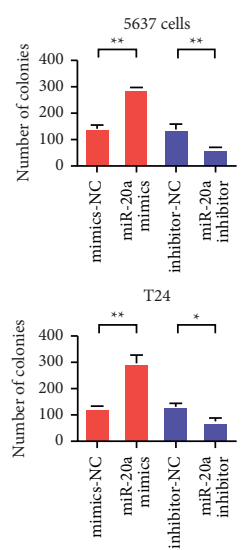

FigURE 2: MiR-20a-5p overexpression promotes the malignant progression of bladder cancer in the 5637 and T24 cells. (a) Detection of miR-20a-5p expression efficiency in the 5637 cells. (b) Detection of miR-20a-5p expression efficiency in the T24 cells. (c) The 5637 cell proliferation assay. (d) The T24 cell proliferation test. (e) The 5637 and T24 cell Transwell tests. (f) The 5637 cell and T24 scratch detection. (g) The 5637 cell and T24 clone formation ability test. ${ }^{*} P<0.05,{ }^{* *} P<0.01$, and magnification is $200 \times$. 


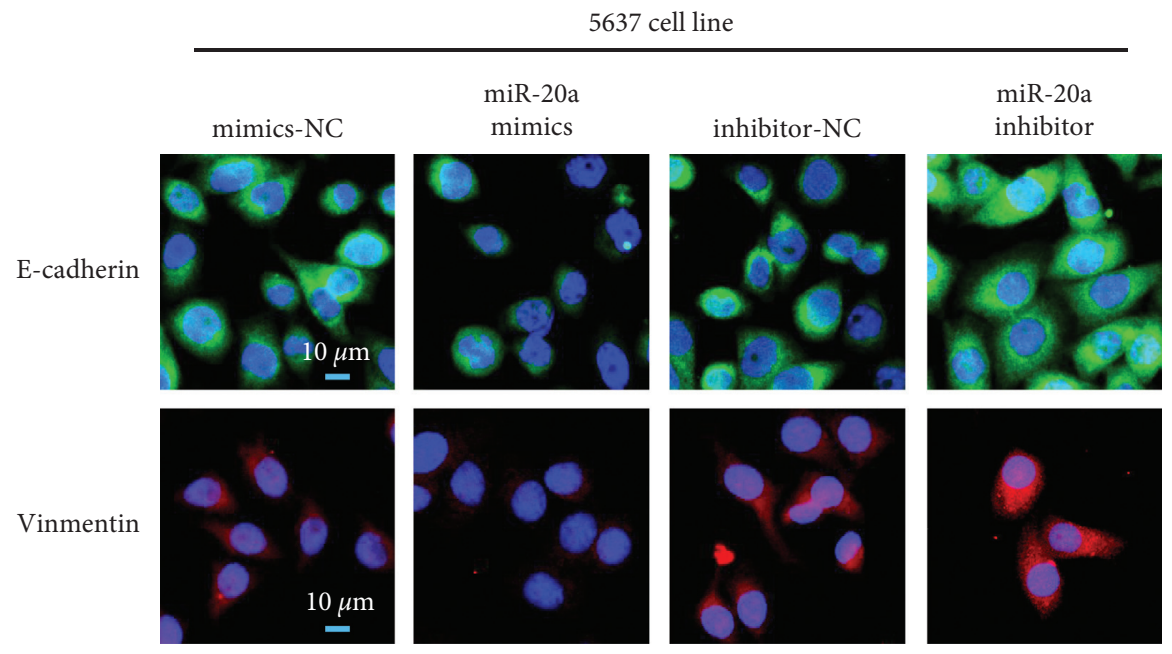

(a)

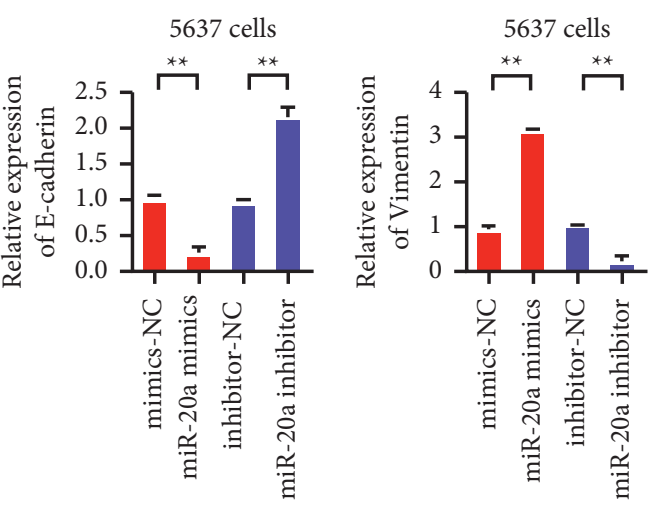

(b)
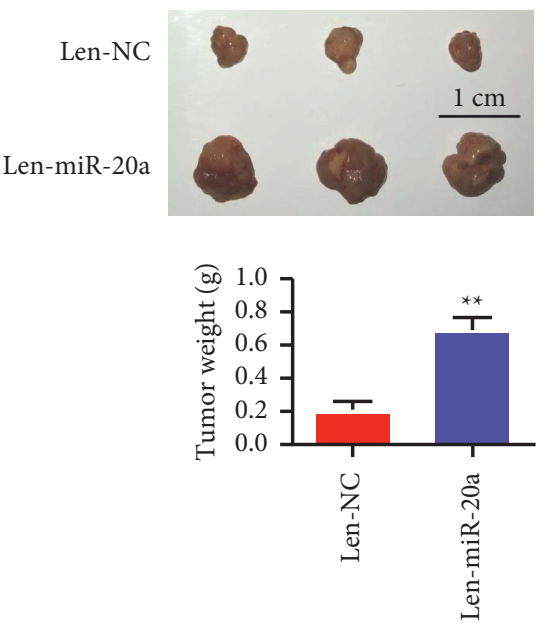

(d)
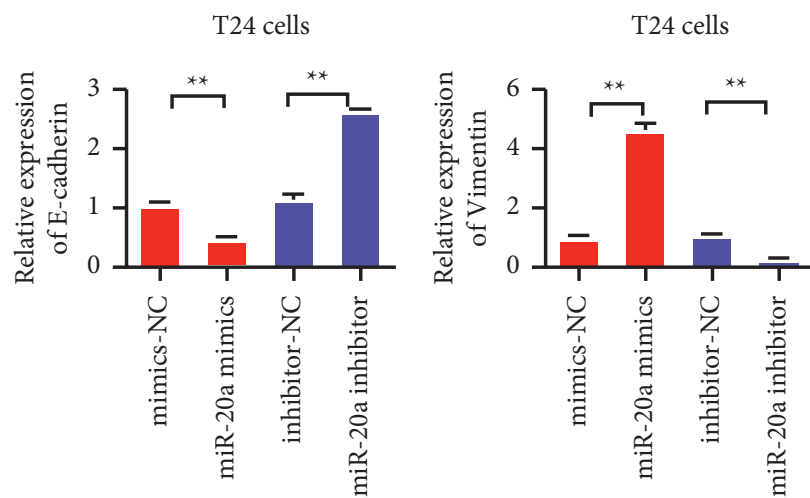

(c)
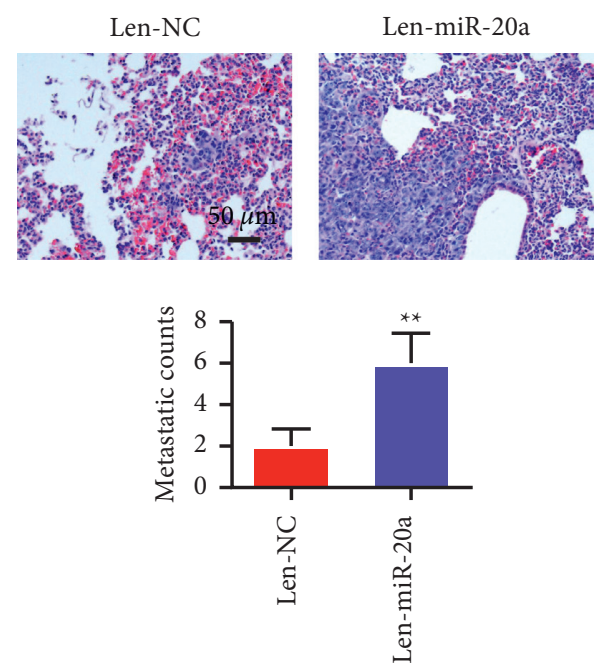

(e)

FIGURE 3: MiR-20a-5p overexpression promotes bladder cancer in 5637 and T24 EMT. (a) Detection of E-cadherin and Vimentin expressions by immunofluorescence staining in the 5637 cells. (b) Detection of E-cadherin and Vimentin expressions in the 5637 cells by RTqPCR. (c) Detection of E-cadherin and Vimentin expressions in the T24 cells by RT-qPCR. (d) Animal experiments verified the effect of miR-20a. Results showed that miR-20a could promote tumor proliferation. (e) miR-20a overexpression promotes tumor metastasis. ${ }^{*} P<0.05,{ }^{* *} P<0.01$, and magnification is $400 \times$. 


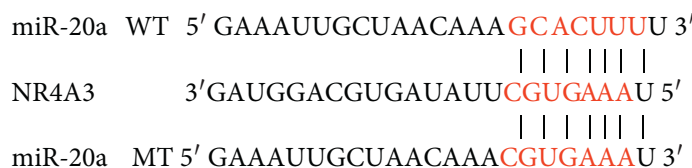

(a)

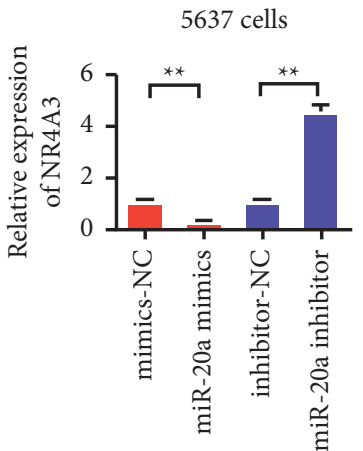

(c)

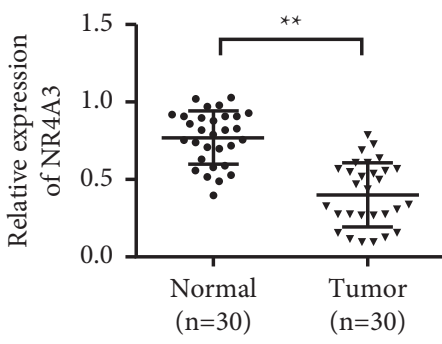

(e)

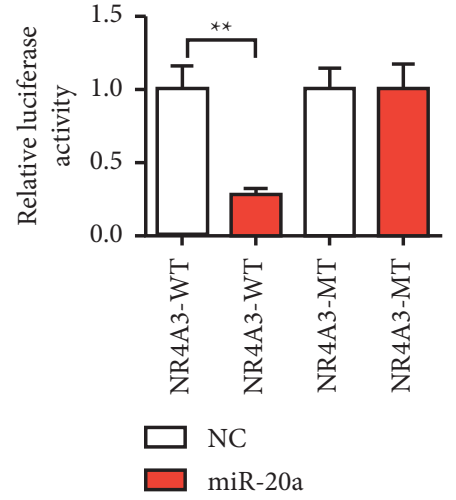

(b)

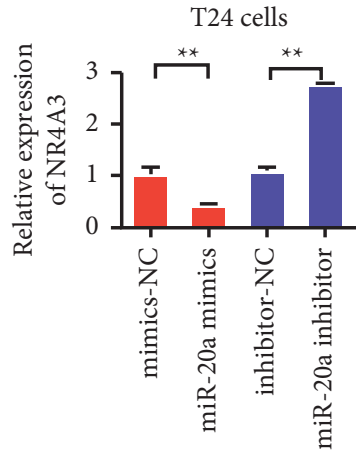

(d)

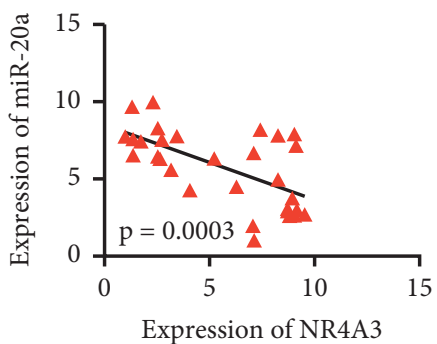

(f)

Figure 4: MiR-20a-5p targeted binding to NR4A3 (a). Information map of miR-20a-5p and NR4A3 binding sites. (b) Dual luciferase reporter gene demonstrates the binding of miR-20a-5p to NR4A3. (c) The level of 5637 cells confirmed that miR-20a-5p overexpression inhibits NR4A3, and miR-20a-5p inhibition upregulates NR4A3. (d) T24 cell level confirmed that miR-20a-5p overexpression inhibits NR4A3, and inhibiting miR-20a-5p upregulates NR4A3. (e) Detection of NR4A3 expression in bladder cancer. (f) Detection of correlation between miR-20a-5p and NR4A3 coexpression. ${ }^{* *} P<0.01$.

structural characteristics and are associated with cell mitogenic reactions. These nuclear receptors are transcription factors that enhance their function by inducing the activation of downstream pathways. They have multiple roles in cell growth and apoptosis pathways [37]. NR4A3 is an orphan nuclear receptor that regulates the transcription of the overlapping target genes. Its transcriptional regulatory activity does not depend on ligands but is regulated by cellspecific and stimulus-specific gene induction and protein phosphorylation, which are related to the signal transduction of hormones, inflammation, mitosis promotion, apoptosis, differentiation, and other cellular processes. It may act as a balance regulator of proliferation, apoptosis, and differentiation and may be related to the sensitivity of cells to tumorigenesis $[4,38]$. NR4A3 is reportedly a tumor suppressor gene in the development of myeloid leukemia. Studies in the mouse models have shown that the deletion of Nur77 and NR4A3 rapidly leads to the development of acute myeloid leukemia.

In this study, bioinformatics methods predicted that NR4A3 was a potential target gene for miR-20a. Fluorescence reporter vector experiments showed that the increased expression of miR-20a in the cells transfected with miR-20a mimics reduced the fluorescence intensity of the $3^{\prime} \mathrm{UTR}$ of NR4A3, and the effect was eliminated after some base mutations at the binding site. A qPCR experiment also confirmed that NR4A3 expression decreased with miR-20a overexpression. When the expression of miR-20a was 


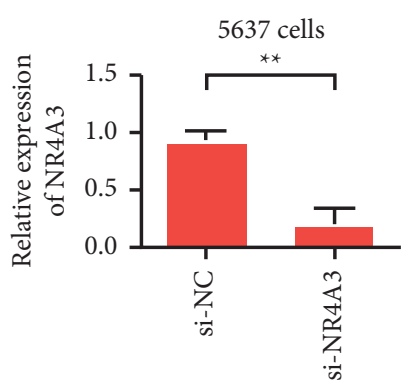

(a)

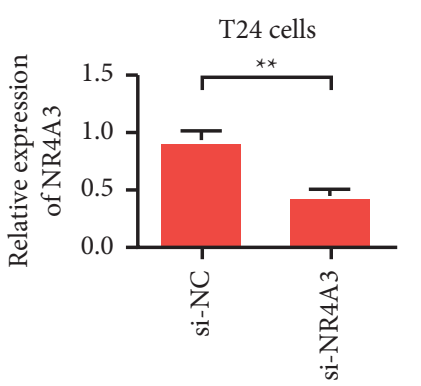

(b)

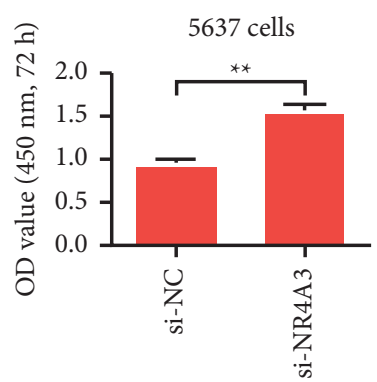

(c)

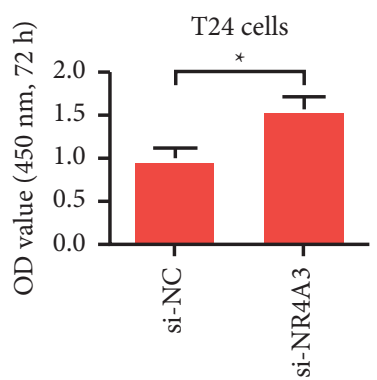

(d)
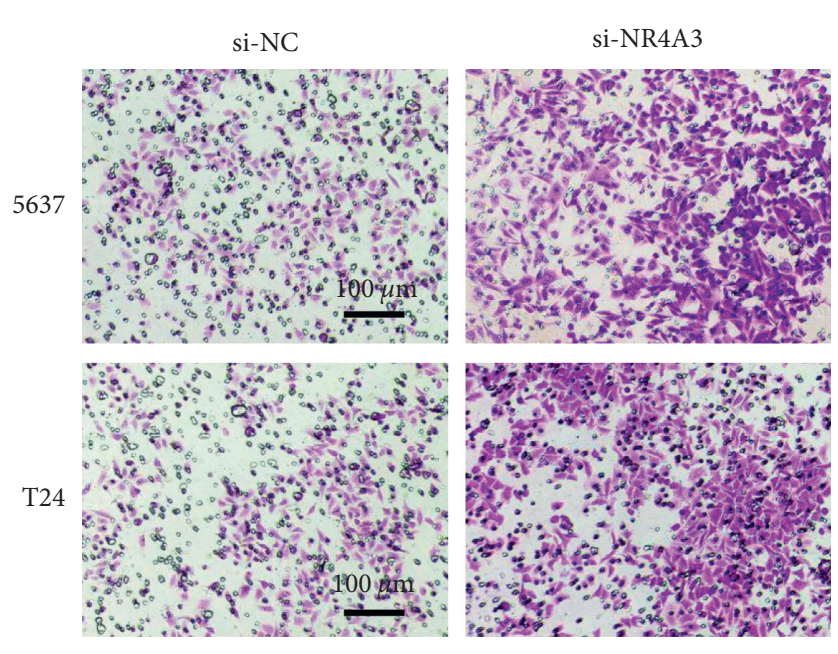

(e)
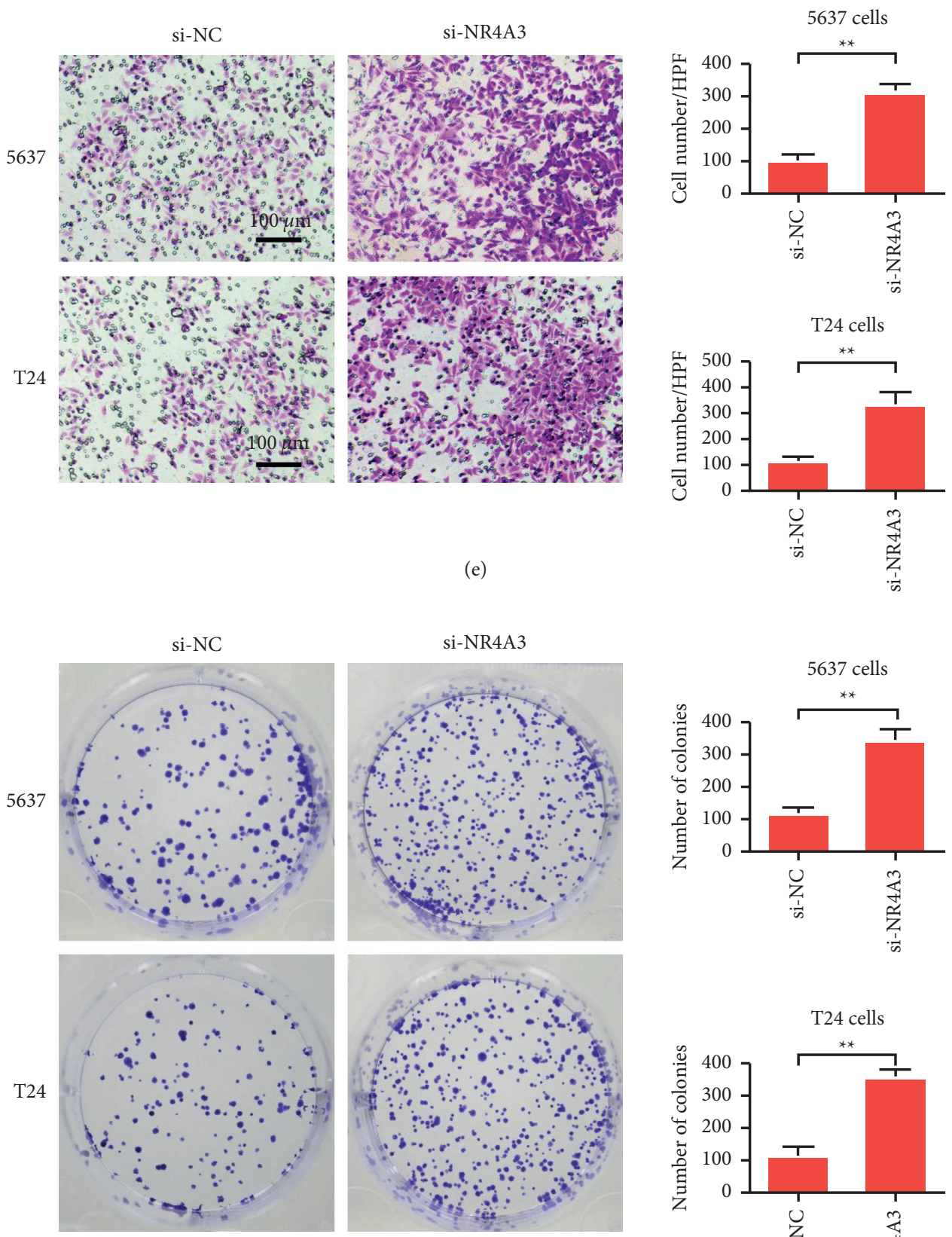

(f)

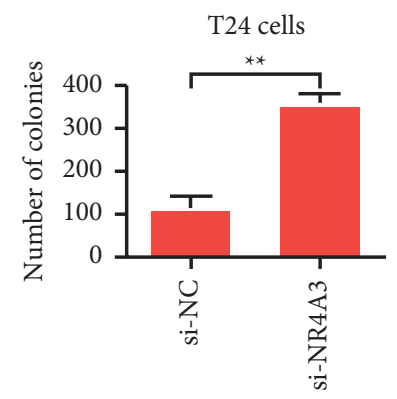

FIGURE 5: Knockdown of NR4A3 promotes the malignant evolution of bladder cells (a) Detection of NR4A3 expression in 5637 cells. (b) Detection of NR4A3 expression in T24 cells. (c) 5637 cell proliferation assay. (d) T24 cell proliferation test. (e) 5637 and T24 cell Transwell assay. (f) 5637 and T24 cell clone formation assay. ${ }^{*} P<0.05,{ }^{* *} P<0.01$, and magnification is $200 \times$. 


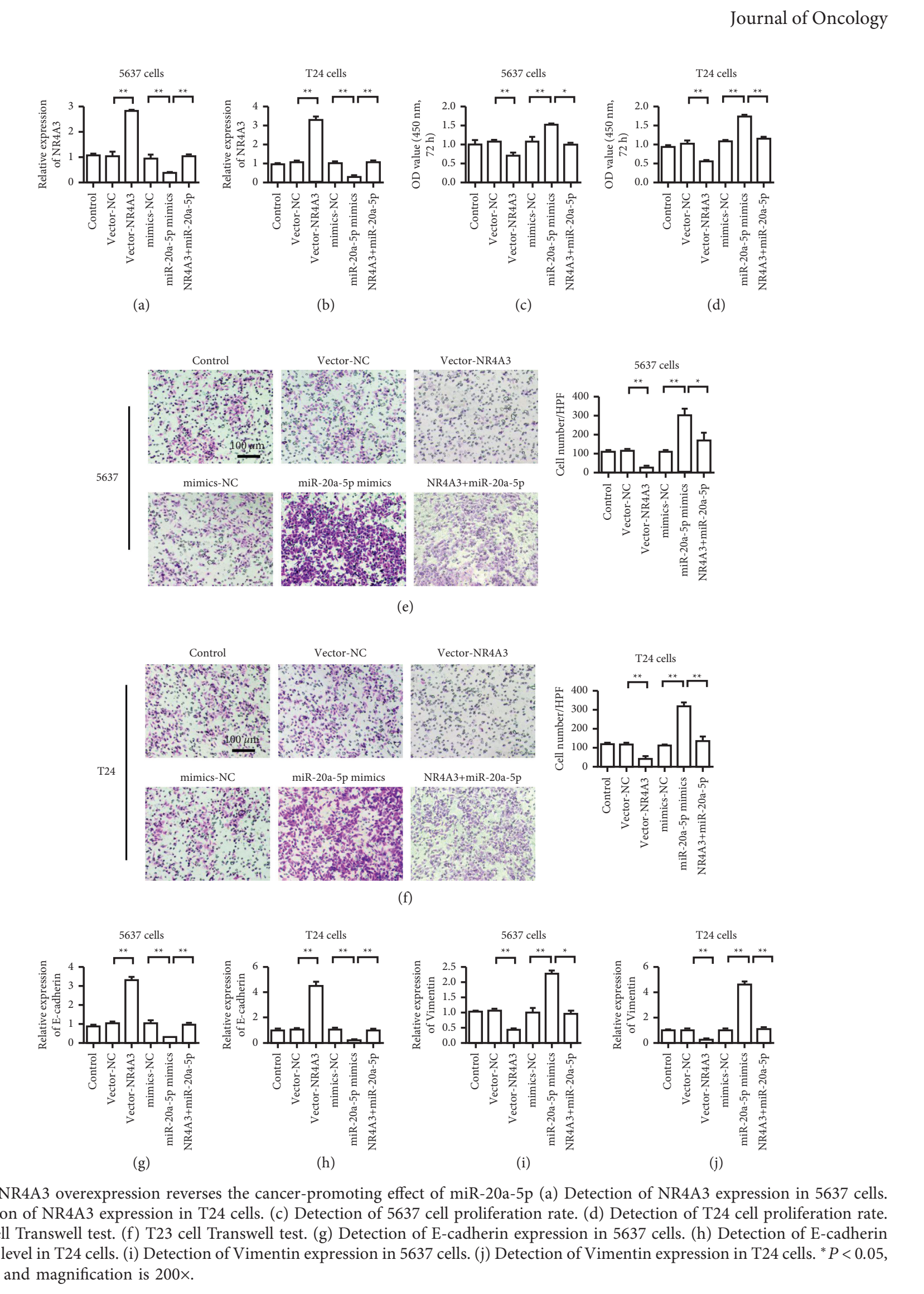


inhibited, the protein level of NR4A3 was upregulated, thereby confirming that NR4A3 was the direct target gene of miR-20a. MiR-20a inhibited NR4A3 expression by acting on the binding sites on the $3^{\prime}$ UTR of NR4A3.

The results of this study showed that the high expression of miR-20a upregulated the expression of mesenchymal marker Vimentin, while it inhibited the expression of the epithelial marker E-cadherin. It suggested that miR-20a could induce EMT. Importantly, we further demonstrate that miR-20a promotes EMT, migration, invasion, and metastasis of the bladder cancer cells by targeting NR4A3. The results of Zhao et al. [39] showed that, in breast cancer, the high expression of NR4A3 inhibits the MEK pathway, and it can inhibit ERK phosphorylation and inhibit the expression of Slug. Therefore, in bladder cancer, miR-20a may activate the MEK signaling pathway by inhibiting NR4A3, thereby promoting EMT and promoting the proliferation and metastasis of bladder cancer.

\section{Conclusion}

We found that miR-20a can promote the invasion of bladder cancer cells and play the role of an oncogene. The mechanism studies showed that the carcinogenic effect of miR-20a was achieved by the inhibition of NR4A3. Therefore, miR20a and NR4A3 may be potential drug therapeutic targets.

\section{Data Availability}

The analyzed datasets generated during the study are available from the corresponding author on reasonable request.

\section{Ethical Approval}

Animal experiments conformed to internationally accepted standards and have been approved by the Ethics Committee of Qujing No. 1 People's Hospital.

\section{Consent}

All patients signed informed consent.

\section{Conflicts of Interest}

The authors declare that they have no conflicts of interest.

\section{Authors' Contributions}

SJM carried out the conception and design and was the guarantor of integrity of the entire study. DLG performed the clinical studies. GY carried out the collection and assembly of data. All authors read and approved the final manuscript.

\section{Supplementary Materials}

Figure S1. The expression of NR4A3 using the UALCAN website. The results showed that NR4A3 was downregulated in bladder cancer. (Supplementary Materials)

\section{References}

[1] X. Zhao, J. Li, S. Huang, X. Wan, H. Luo, and D. Wu, "MiRNA-29c regulates cell growth and invasion by targeting CDK6 in bladder cancer," American Journal of Tourism Research, vol. 7, pp. 1382-1389, 2015.

[2] X. H. Yin, Y. H. Jin, Y. Cao et al., "Development of a 21-miRNA signature associated with the prognosis of patients with bladder cancer," Frontiers in Oncology, vol. 9, p. 729, 2019.

[3] L. Xueyong and L. Dong, "miRNA-137 expression in bladder cancer and the impact on the invasion and migration of bladder cancer cells," Oncology Progress, 2019.

[4] X. Xi, N. Liu, Q. Wang et al., "ACT001, a novel PAI-1 inhibitor, exerts synergistic effects in combination with cisplatin by inhibiting PI3K/AKT pathway in glioma," Cell Death \& Disease, vol. 10, no. 10, p. 757, 2019.

[5] Y. Wang, Z. Li, Q. Lin et al., "Highly sensitive detection of bladder cancer-related miRNA in urine using time-gated luminescent biochip," ACS Sensors, vol. 4, no. 8, pp. 2124-2130, 2019.

[6] K. Miyamoto, N. Seki, R. Matsushita, H. Yoshino, M. Nakagawa, and H. Enokida, "Tumour-suppressive miRNA-26a-5p and miR-26b-5p inhibit cell aggressiveness by regulating PLOD2 in bladder cancer," British Journal of Cancer, vol. 115, no. 3, 2016.

[7] M. Chen, C. Zhuang, Y. Liu et al., "Tetracycline-inducible shRNA targeting antisense long non-coding RNA HIF1AAS2 represses the malignant phenotypes of bladder cancer," Cancer Letters, vol. 376, no. 1, pp. 155-164, 2016.

[8] M. Chen, J. Li, C. Zhuang, and Z. Cai, "Increased lncRNA ABHD11-AS1 represses the malignant phenotypes of bladder cancer," Oncotarget, vol. 8, no. 17, pp. 28176-28186, 2017.

[9] R. M. Drayton, E. Dudziec, S. Peter et al., "Reduced expression of miRNA-27a modulates cisplatin resistance in bladder cancer by targeting the cystine/glutamate exchanger SLC7A11," Clinical Cancer Research an Official Journal of the American Association for Cancer Research, vol. 20, no. 7, 2014.

[10] N. Ratert, H.-A. Meyer, M. Jung et al., "miRNA profiling identifies candidate miRNAs for bladder cancer diagnosis and clinical outcome," Journal of Molecular Diagnostics, vol. 15, no. 5, pp. 695-705, 2013.

[11] X. Liu, Y. Wu, Z. Zhou et al., "Celecoxib inhibits the epithelialto-mesenchymal transition in bladder cancer via the miRNA145/TGFBR2/Smad3 axis," International Journal of Molecular Medicine, vol. 44, no. 2, 2019.

[12] W. Zhong, W. Yang, Y. Qin et al., "6-gingerol stabilized the p-VEGFR2/VE-cadherin/ $\beta$-catenin/actin complex promotes microvessel normalization and suppresses tumor progression," Journal of Experimental \& Clinical Cancer Research, vol. 38, no. 1, p. 285, 2019.

[13] U. A. Ørom, F. C. Nielsen, and A. H. Lund, "MicroRNA-10a binds the 5' UTR of ribosomal protein mRNAs and enhances their translation," Molecular Cell, vol. 30, pp. 460-471, 2008.

[14] J. E. Fish, M. M. Santoro, S. U. Morton et al., "miR-126 regulates angiogenic signaling and vascular integrity," $D e$ velopmental Cell, vol. 15, no. 2, pp. 272-284, 2008.

[15] G. A. Calin, C. D. Dumitru, M. Shimizu et al., "Nonlinear partial differential equations and applications: frequent deletions and down-regulation of micro-RNA genes miR15 and miR16 at 13q14 in chronic lymphocytic leukemia," Proceedings of the National Academy of Sciences, vol. 99, no. 24, pp. 15524-15529, 2002. 
[16] I.-K. Wang and C.-Y. Li, "miR-20a-5p mediates hypoxiainduced autophagy by targeting ATG16L1 in acute kidney injury," Critical Care, vol. 18, no. S2, p. P15, 2014.

[17] I.-K. Wang, K.-T. Sun, T.-H. Tsai et al., "MiR-20a-5p mediates hypoxia-induced autophagy by targeting ATG16L1 in ischemic kidney injury," Life Sciences, vol. 136, pp. 133-141, 2015.

[18] D. Bluteau, L. Gilles, M. Hilpert et al., "Down-regulation of the RUNX1-target gene NR4A3 contributes to hematopoiesis deregulation in familial platelet disorder/acute myelogenous leukemia," Blood, vol. 118, no. 24, pp. 6310-6320, 2011.

[19] C. J. Broehm, J. Wu, R. R. Gullapalli, and T. Bocklage, "Extraskeletal myxoid chondrosarcoma with a t $(9 ; 16)$ (q22; p11.2) resulting in a NR4A3-FUS fusion," Cancer Genetics, vol. 207, no. 6, pp. 276-280, 2014.

[20] Y. Jeong, Y. Xie, G. Xiao et al., "Nuclear receptor expression defines a set of prognostic biomarkers for lung cancer," PLoS Medicine, vol. 7, no. 12, p. e1000378, 2010.

[21] A. M. Ramirez-Herrick, S. E. Mullican, A. M. Sheehan, and O. M. Conneely, "Reduced NR4A gene dosage leads to mixed myelodysplastic/myeloproliferative neoplasms in mice," Blood, vol. 117, no. 9, pp. 2681-2690, 2011.

[22] R. Borup, M. Rossing, R. Henao et al., "Molecular signatures of thyroid follicular neoplasia," Endocrine-Related Cancer, vol. 17, no. 3, pp. 691-708, 2010.

[23] H. Luo, C. Xu, W. Le, B. Ge, and T. Wang, "lncRNA CASC11 promotes cancer cell proliferation in bladder cancer through miRNA-150," Journal of Cellular Biochemistry, vol. 120, no. 8, 2019.

[24] H. Hiroshi, U. Koji, S. Varahram et al., "Oncogenic miRNA182-5p targets Smad4 and RECK in human bladder cancer," PLoS One, vol. 7, p. e51056, 2012.

[25] F. Gottardo, C. G. Liu, M. Ferracin et al., "Micro-RNA profiling in kidney and bladder cancers," Urologic Oncology: Seminars and Original Investigations, vol. 25, no. 5, pp. 387-392, 2007.

[26] J. W. F. Catto, S. Miah, H. C. Owen et al., "Distinct MicroRNA alterations characterize high-and low-grade bladder cancer," Cancer Research, vol. 69, no. 21, pp. 8472-8481, 2009.

[27] C. Blick, A. Ramachandran, R. Mccormick et al., "Identification of a hypoxia-regulated miRNA signature in bladder cancer and a role for miR-145 in hypoxia-dependent apoptosis," British Journal of Cancer, vol. 113, no. 4, 2015.

[28] A. Enkelmann, J. Heinzelmann, F. Von Eggeling et al., "Specific protein and miRNA patterns characterise tumourassociated fibroblasts in bladder cancer," Journal of Cancer Research and Clinical Oncology, vol. 137, no. 5, 2011.

[29] X. Xi, Y. Chu, N. Liu et al., "Joint bioinformatics analysis of underlying potential functions of hsa-let-7b-5p and core genes in human glioma," Journal of Translational Medicine, vol. 17, no. 1, p. 129, 2019.

[30] H. Yoshino, N. Seki, T. Itesako, T. Chiyomaru, M. Nakagawa, and H. Enokida, "Aberrant expression of microRNAs in bladder cancer," Nature Reviews Urology, vol. 10, no. 7, pp. 396-404, 2013.

[31] Y. Chang, C. Liu, J. Yang et al., "MiR-20a triggers metastasis of gallbladder carcinoma," Journal of Hepatology, vol. 59, no. 3, pp. 518-527, 2013.

[32] G. Huang, K. Nishimoto, Z. Zhou, D. Hughes, and E. S. Kleinerman, "miR-20a encoded by the miR-17-92 cluster increases the metastatic potential of osteosarcoma cells by regulating Fas expression," Cancer Research, vol. 72, no. 4, pp. 908-916, 2011.
[33] Q. Xu, Q.-G. Dong, L.-p. Sun, C.-y. He, and Y. Yuan, "Expression of serum miR-20a-5p, let-7a, and miR-320a and their correlations with pepsinogen in atrophic gastritis and gastric cancer: a case-control study," BMC Clinical Pathology, vol. 13, no. 1, p. 11, 2013.

[34] T.-M. Yu, I. K. Wang, K. T. Sun et al., “MiR-20a-5p mediates hypoxia-induced autophagy by targeting ATG16L1 in ischemic kidney injury," Life Sciences, vol. 136, 2015.

[35] S. Kagaya, N. Ohkura, T. Tsukada et al., "Prostaglandin A2 acts as a transactivator for NOR1 (NR4A3) within the nuclear receptor superfamily," Biological and Pharmaceutical Bulletin, vol. 28, no. 9, pp. 1603-1607, 2005.

[36] S. E. Mullican, S. Zhang, M. Konopleva et al., "Abrogation of nuclear receptors Nr4a3 andNr4a1 leads to development of acute myeloid leukemia," Nature Medicine, vol. 13, no. 6, pp. 730-735, 2007.

[37] Q. X. Li, N. Ke, R. Sundaram, and F. Wong-Staal, "NR4A1, 2, 3 an orphan nuclear hormone receptor family involved in cell apoptosis and carcinogenesis," Histology Histopathology, vol. 21 , no. $5,2006$.

[38] W. Zhong, H. Hou, T. Liu et al., "Cartilage oligomeric matrix protein promotes epithelial-mesenchymal transition by interacting with transgelin in colorectal cancer," Theranostics, vol. 10, no. 19, pp. 8790-8806, 2020.

[39] X. G. Zhao, J. Y. Hu, J. Tang et al., "miR-665 expression predicts poor survival and promotes tumor metastasis by targeting NR4A3 in breast cancer," Cell Death \& Disease, vol. 10, p. 479, 2019. 